\title{
Avaliação funcional de neutrófilos sanguíneos em vacas leiteiras infectadas pelo vírus da leucemia bovina
}

José Augusto Ferronatto[a, $]^{[}$, Maiara Garcia Blagitz ${ }^{[b]}$, Fernando Nogueira de Souza ${ }^{[b]}$, Camila Freitas Batista ${ }^{[b]}$, Luis Fernando Fernandes Azevedo ${ }^{[b]}$,Alice Maria Melville Paiva Della Libera ${ }^{[b]}$

\footnotetext{
[a] Universidade do Oeste de Santa Catarina (UNOESC), Xanxerê, SC, Brasil

[b] Veterinary Clinical Immunology Research Group, Departamento de Clínica Médica, Faculdade de Medicina Veterinária e Zootecnia, Universidade de São Paulo (USP), São Paulo, SP, Brasil
}

${ }^{*}$ Autor correspondente

e-mail: jose_augustoferronatto@hotmail.com

\section{Resumo}

Os neutrófilos têm participação crucial na defesa contra diversos micro-organismos, sendo conhecidos como células fagocíticas. Sabe-se que as doenças virais podem afetar funções tanto do sistema imune inato quanto do adaptativo, o que pode predispor os animais a coinfecções. Portanto, o presente estudo objetivou avaliar a função dos neutrófilos sanguíneos e perfil de linfócitos em vacas leiteiras naturalmente infectadas pelo vírus da leucemia bovina (VLB) com e sem linfocitose persistente (LP). Para isto, o presente estudo utilizou 25 vacas Holandesas que foram separadas em três grupos: 1 - vacas não infectadas pelo VLB (resultados negativos nos testes de imunodifusão em ágar gel e ELISA para o antígeno gp51 do VLB) e sem alterações hematológicas $(\mathrm{n}=9$ ); 2 - vacas infectadas pelo VLB (resultados positivos nos testes de imunodifusão em ágar gel e ELISA para o antígeno gp51 do VLB) e sem alterações hematológicas [alinfocitóticos (AL), n = 10]; e 3 - vacas infectadas pelo VLB com LP $(n=6)$. Após 110 dias da primeira amostragem, novas coletas foram feitas para confirmar a LP, além de coleta de amostras de sangue para determinar a fagocitose de Staphylococcus aureus, produção intracelular de espécies reativas de oxigênio (ERO), viabilidade e expressão de moléculas de adesão em neutrófilos por citometria de fluxo. Os resultados obtidos demonstraram que as vacas infectadas pelo VLB com PL apresentaram alterações nas funções dos neutrófilos, como menor porcentagem de neutrófilos que produziram espécies reativas de oxigênio (ERO; 68,25 \pm 4,39\%) e fagocitaram S. aureus (58,00 $\pm 10,62 \%$ ) do que os bovinos não infectados pelo VLB (ERO: 94,80 \pm 1,76\%; Fagocitose: 79,70 \pm 4,03\%). A percentagem relativa de neutrófilos que expressaram CD44 (78,83 $\pm 2,88 \%)$ e CD11b (61,35 $\pm 6,88 \%)$ foi menor em vacas infectadas com BLV manifestando PL, que nos animais sadios não infectados (CD44+: 96,13 \pm 0,85 \%; $\left.\mathrm{CD}_{11} \mathrm{~b}^{+}: 84,88 \pm 6,01 \%\right)$ e animais infectados sem LP (CD44+: 92,21 $\pm 1,15 \%$; CD11b: 88,18 $\left.\pm 3,67 \%\right)$. No 
entanto, não se observou diferença significativa na expressão de CD62L pelos neutrófilos. Foi encontrada correlação entre os neutrófilos que expressaram CD11b e os que produziram ERO ( $r=0,67 ; \mathrm{P}=0,0003$ ). Além disso, a percentagem de neutrófilos $\mathrm{CD} 44^{+}$foi positivamente correlacionada com a percentagem de neutrófilos que fagocitaram $S$. aureus $(\mathrm{r}=0,39 ; \mathrm{P}=0,05)$. Observou-se, também, inibição da apoptose de neutrófilos em animais infectados pelo VLB, particularmente aqueles com LP. Ademais, foi observada uma correlação positiva entre a percentagem relativa de neutrófilos $\mathrm{CD}_{11} \mathrm{~b}^{+}$e a proporção de neutrófilos produzindo ERO em bovinos leiteiros. Por fim, a infecção pelo VLB, particularmente em vacas infectadas manifestando PL, pode predispor a doenças secundárias. 